\title{
Aprender a estudiar textos en primaria: propuestas para innovar en las prácticas de lectura de textos escolares
}

\author{
Learning to Study Texts: Innovative Activities to \\ Enrich Textbook Reading Practices in Elementary \\ Education
}

\section{Angélica Sepúlveda ${ }^{1}$, Nicole Paulet Piedra ${ }^{2}$ y Beatriz Cardoso ${ }^{2}$}

${ }^{1}$ Universidade do Estado de Mato Grosso

${ }^{2}$ Laboratório de educação (ONG, Brasil)

\section{Resumen}

\begin{abstract}
Aprender a estudiar textos es un programa de innovación educativa que busca mejorar la enseñanza y el aprendizaje de contenidos disciplinares a partir de propuestas didácticas basadas en el análisis y el comentario del lenguaje de los textos. Este artículo presenta las bases del programa y un estudio piloto realizado con dos profesoras de $4^{\circ}$ año de primaria que implementaron durante cuatro meses las propuestas del programa en clases de Historia. Los análisis incluyeron la comparación de las prácticas docentes en torno de la lectura de textos escolares de historia antes y después de conocer Aprender a estudiar textos y también la descripción del modo en que fueron cambiando las prácticas a lo largo de la intervención. Los resultados indican que, con la incorporación de las propuestas del programa, las profesoras pasaron a ofrecer más oportunidades a los alumnos para atender, recuperar, comentar y analizar el contenido informativo de los textos. La principal contribución del estudio consistió en ofrecer insumos para el diseño de un programa de formación permanente de docentes.
\end{abstract}

Palabras clave: lenguaje académico; lectura; educación primaria; prácticas docentes

Correspondencia a:

Angélica Sepúlveda

Rua Artemio Zonta, 61. CP: 78345-000, Castanheira, Mato Grosso, Brasil. luz.angelica@unemat.edu

(c) 2021 PEL, http://www.pensamientoeducativo.org - http://www.pel.cl 


\title{
Abstract
}

\begin{abstract}
Learning to Study Texts is an innovative program that seeks to improve teaching and learning of disciplinary content by using teaching proposals based on analyzing and commenting on the language used in texts. This paper presents the bases of the program and a pilot study conducted with two teachers working in the fourth grade of elementary school who implemented the program proposals in History classes for four months. This analysis included the comparison of teaching practices involved in reading History texts before and after following the Learning to Study Texts program. It also included the description of how they changed their practices throughout the intervention. The results indicate that, with the incorporation of the program proposals, the teachers began to provide more opportunities to the students to listen, retrieve, comment on and analyze the information content of the texts. The main contribution of this study is to offer inputs for the design of a continuous teacher training program..
\end{abstract}

Keywords: academic language; reading; elementary school; teaching practices

\section{Introducción}

El lenguaje utilizado en los textos escolares ha sido descrito y analizado por numerosos lingüistas sistémicofuncionales (Halliday \& Martin, 1993; Martin \& Rose, 2008; Moss et al., 1998; Schleppegrell, 2001, 2004). Las investigaciones sobre los desafíos que supone su lectura destacan la alta proporción de nombres y de grupos nominales (Fang, Schleppegrell \& Cox, 2006; Schleppegrell, 2001) con la inclusión de términos técnicos, numerosas palabras abstractas, metáfora gramatical, entre otros.

Se ha señalado que mientras en los usos cotidianos del lenguaje suele existir una proporción equilibrada entre la presencia de nombres y verbos, en el lenguaje de los textos escolares hay una mayor concentración de nombres (Halliday \& Martin, 1993), lo que aumenta la densidad informativa. Al condensar un número elevado de información en pocas palabras, los lectores precisan restituir y comprender más ideas para cada segmento textual (Fang et al., 2006).

Otro aspecto relacionado con la condensación de la información en los textos escolares se manifiesta en el uso no prototípico de ciertas estructuras gramaticales; por ejemplo, el uso de nombres para expresar procesos o propiedades (colonización de colonizar, impopularidad de impopular), fenómeno conocido como nominalización (Moss, Barletta, Chamorro \& Mizuno, 2013). Las nominalizaciones expresan mediante una única palabra (sustantivo) aquello que cotidianamente exigiría explicitar participantes, procesos y circunstancias. En estos casos, los lectores necesitan recuperar la información condensada, restituyendo, por ejemplo, los actores responsables de los procesos o propiedades explicadas (quién colonizó qué, quién era impopular dónde).

Estas demandas están presentes desde los años finales de la educación primaria, cuando los textos escolares comienzan a exhibir las características propias del lenguaje académico (Biemiller, 1999; Fang et al., 2006). Los estudiosos de estos fenómenos señalan que son necesarias intervenciones didácticas específicas, a través de las asignaturas y grados escolares, para ayudar a los alumnos a lidiar con la lengua propia de las diferentes disciplinas y realizar actividades que promuevan el aprendizaje de contenidos curriculares y de las formas de comunicación académica (Fang \& Schleppegrell, 2008; Moss, 2006, 2009; Moss, et al., 2013; Schleppegrell, 2001, 2004; Uccelli, Phillips Galloway, Aguilar \& Allen, 2019). 
Las investigaciones educativas sobre el aprendizaje a partir de la lectura de textos escolares documentan que los alumnos no reciben las ayudas necesarias para enfrentar con éxito este tipo de desafío (Aisenberg, 2007; Bortoni-Ricardo, 2008; Chamorro, Barletta \& Mizuno, 2013; Moss, 2006, 2009; Moss, et al., 2013; Natale, 2009; Pereira, 2007). A lo largo de la escolaridad, la falta de comprensión y apropiación del discurso expositivo, informativo y científico propio del lenguaje académico crea barreras para acceder al conocimiento disciplinar (Halliday, 1998) y se ha revelado como un factor asociado con los niveles de desempeño en tareas de comprensión de lectura y producción escrita (Meneses et al., 2018; Phillips Galloway \& Uccelli, 2019a, 2019b; Uccelli, Galloway, Barr, Meneses \& Dobbs, 2015).

El programa Aprender a estudiar textos (en adelante AET) promueve cambios en las prácticas pedagógicas para responder a los desafíos propios de la enseñanza de contenidos disciplinares a partir de la lectura de textos. Con este objetivo, desarrollamos secuencias didácticas modelo ${ }^{1}$ basadas en la atención, anotación y manipulación de la disposición gráfica del texto. A través de estos modelos, se presentan y analizan las características del lenguaje de textos escolares y se proponen secuencias de actividades de lectura, análisis y representación de su contenido informativo. La aproximación y el conjunto de actividades del programa son aplicables a diferentes áreas del currículo. En este trabajo nos centramos en el área de Historia. ${ }^{2}$ Por esto, antes de presentar las propuestas de prácticas pedagógicas específicas del programa, nos detenemos en la caracterización de algunos de los desafíos propios de la lectura de los textos escolares de esta área y en los tipos de actividades y materiales educativos que han sido propuestos para su estudio.

\section{Las características del lenguaje de los textos escolares de Historia}

Los textos escritos para narrar la historia de pueblos y civilizaciones localizan en el tiempo y el espacio eventos del pasado. Los lectores de estos textos precisan ser capaces de identificar informaciones clave relativas a los participantes, acontecimientos y circunstancias relatadas de lugar, tiempo, causa, modo, entre otras (Schleppegrell, Achugar \& Oteíza, 2004). Es decir, deben ser capaces de identificar explícitamente en un texto quién hizo qué, a quién y en qué circunstancias. Por esto, para el área de Historia, AET propone que durante el estudio de los textos se atienda a las palabras que expresan tales informaciones.

La literatura especializada ha destacado fenómenos relacionados con las palabras utilizadas en los textos para presentar los acontecimientos y los participantes, y aquellas que caracterizan el tipo de organización informativa predominante. Las palabras utilizadas para presentar los acontecimientos relatados son los verbos, por lo general, en pretérito. Siguiendo la clasificación de Schleppegrell y sus colegas (2004), los verbos que con más frecuencia se utilizan para escribir sobre la Historia son verbos de acción como luchar, partir, extraer; verbos de decir y pensar-sentir como anunciar, creer, sufrir, y verbos de ligación como ser, estar, tener. Las autoras destacan que esta categorización permite diferenciar entre el relato de eventos, la expresión de actitudes u opiniones y la descripción de información contextual. En cuanto a los verbos de decir-pensar-sentir, se ha señalado que su uso más frecuente en los textos escolares no consiste en expresar comentarios, análisis u opiniones de los autores sobre los contenidos históricos, sino en expresar los sentimientos, pensamientos o actos de habla de los participantes de la historia (Moss, 2000).

1. AET no es un currículum. Las secuencias didácticas que componen el material del programa son un modelo a partir del cual se introducen las características del lenguaje de los textos escolares de Historia y los tipos de actividad que pueden realizarse con los alumnos.

2. La perspectiva de las autoras es validar el abordaje de AET en Historia para más adelante retomar la producción de contenidos ajustados a las características propias de otras áreas del conocimiento, como Geografía o Ciencias. 
Las palabras utilizadas para aludir a los participantes son nombres y grupos nominales que indican sobre quién o sobre qué es el contenido relatado, descrito o explicado en cada enunciado del texto. Estos nombres varían en un continuo que se mueve entre términos específicos con referencia objetiva en el mundo físico a otros abstractos que corresponden a conceptos, relaciones o procesos (Martin \& Rose, 2008; Schleppegrell, 2001). Así, por ejemplo, se utilizan nombres propios para referirse a participantes humanos, individuos específicos como Pêro Vaz de Caminha, colectivos como los trabajadores, los colonizadores, a participantes no humanos, concretos como el petróleo o abstractos como la esclavitud, la independencia. Este último tipo de participantes expresados en nominalizaciones ha recibido bastante atención por parte de los investigadores (Moss et al., 2013). Las nominalizaciones presentan fenómenos como estados o circunstancias dadas, sin agencia humana identificable. Se ha señalado que, si bien cumplen un importante papel en la organización y comunicación del discurso científico (Schleppegrell, 2001), resultan desafiantes para los lectores escolares que precisan, en el caso de los textos de Historia, restituir los agentes responsables por los sucesos o procesos informados (Moss et al., 2013), identificar quién está por detrás de lo ocurrido (Schleppegrell et al., 2004).

En cuanto a las palabras que ayudan a identificar el tipo de organización predominante de los textos escolares de Historia, se encuentran aquellas que indican relaciones temporales y causales entre los acontecimientos. Los análisis de Coffin $(1997,2006)$ han establecido que los textos que comunican la Historia se mueven de la narración lineal de eventos del pasado a la exposición de la Historia como explicaciones o argumentos con foco en tesis abstractas organizadas retóricamente en el tiempo del texto. A grandes rasgos, se espera que los alumnos transiten desde textos caracterizados por el recuento de eventos en secuencia, organizados mediante expresiones y conectores temporales, a textos explicativos en los que predominan vínculos causales, para después leer textos argumentativos, organizados retóricamente, que discuten y debaten hechos del pasado.

\section{Actividades y materiales educativos para conocer el lenguaje de los textos}

Los estudiosos de las características del lenguaje de los textos escolares resaltan la necesidad de que profesores y alumnos reflexionen sobre los desafíos comprensivos propios de los textos académicos (Barletta \& Mizuno, 2005; Fang \& Schleppegrell, 2008, 2010; Moss, 2006, 2009; Schleppegrell \& Achugar, 2003; Schleppegrell et al., 2004; Schleppegrell \& Oliveira, 2006). En sus trabajos de intervención en educación secundaria, estos autores enseñan a restituir la información que el texto omite, modelan formas de ayudar a los alumnos a cuestionar la información que presenta el texto, especialmente a indagar acerca de los participantes humanos de los eventos, sus intereses, roles y motivaciones.

En educación primaria, las propuestas de Teberosky (Ortega, Coromina \& Teberosky, 2013; Teberosky, 2011, 2020; Teberosky, Ortega \& Coromina, 2017; Teberosky \& Sepúlveda, 2011) suman al análisis funcional de los textos secuencias de tareas encadenadas que aprovechan diversas posibilidades de visualización y manipulación del material textual. Las tareas encadenadas consisten en secuencias de actividades interdependientes, que incluyen la lectura en voz alta de la profesora dirigida a los nińos, la visualización, comentario, anotación y etiquetado de la estructura y contenido informativo del texto, la manipulación del léxico del texto y la producción de representaciones de diversos tipos.

En cuanto a la manipulación del material textual, con la intención de favorecer y apoyar los procesos de atención, comentario y análisis del lenguaje de los textos, en estos trabajos (Ortega et al., 2013; Teberosky, Ortega, \& Coromina, 2017; Teberosky \& Sepúlveda, 2011) se propone recuperar un tipo de presentación visual del texto basada en una segmentación textual antigua, conocida como per cola et commata. Este tipo de segmentación hace coincidir la línea con una unidad de sentido (Blanche-Benveniste, 2008). El texto lineal reformateado per cola et commata pasa a consistir en secuencias textuales cortas que coinciden con unidades de lectura semántica 
y unidades de respiración (según la explicación de Johannot, citada por Blanche-Benveniste, 2008). En las intervenciones didácticas referidas se destaca que este formato de presentación resulta útil para las actividades de estudio de los textos (ver figura 1).

Otra de las propuestas consiste en promover la manipulación del léxico del texto (Ortega et al., 2013). Dispuestas las palabras de contenido en etiquetas manipulables, orientan a los alumnos a colocarlas en relación (ver figura 2). Según la jerarquía de la información del texto, los alumnos pueden establecer relaciones de coordinación entre las etiquetas o de categorización. En las observaciones realizadas en estos trabajos (Ortega, 2013), la manipulación del léxico del texto ofrece a los alumnos oportunidades para revisar su comprensión y familiarizarse con un procedimiento de representación esquemática de la información textual.

En AET, este tipo de intervenciones son propuestas a profesores y alumnos de los últimos años de educación primaria $^{3}$ con la intención tanto de aumentar las oportunidades de aprendizaje de contenidos disciplinares a partir de la lectura de textos escolares, como de promover el conocimiento y apropiación de los usos del lenguaje propios de la representación y comunicación de conocimiento disciplinar.

\section{Aprender a estudiar textos y la promoción de cambios en las prácticas pedagógicas}

Las actividades que conforman AET están organizadas en cuatro bloques que responden a diferentes finalidades y momentos de interacción de los alumnos con los textos de estudio (Laboratório de educação, 2019). El primero, preparar a los alumnos para estudiar el texto, incluye diversas acciones que pueden realizarse para explicitar los objetivos de la lectura, activar conocimientos previos, estimular la formación de expectativas y orientar en general la recepción del texto. El segundo, leer y comentar el texto, propone una lectura en voz alta, expresiva e intencionada de la profesora dirigida a los alumnos; orienta a la recuperación del diálogo iniciado antes de la lectura del texto, identificando los temas abordados y estableciendo la necesidad de relectura y estudio del texto. El tercer bloque, estudiar el texto, presenta diversas actividades que pueden ser realizadas para identificar con detalle el contenido informativo del texto (quién hizo qué a quién, bajo qué circunstancias) y reparar en su estructura discursiva (secuencias textuales, cadenas referenciales, marcadores discursivos) (ver figura 1). En este mismo bloque se proponen actividades para estudiar el vocabulario del texto (ver figura 2). Finalmente, el cuarto bloque, comunicar la comprensión del texto, se centra en actividades de comunicación oral y escrita de las comprensiones obtenidas mediante representaciones de diversos tipos.

3. El contenido del programa responde a desafíos enfrentados en el $4^{\circ}$ y $5^{\circ}$ año. Sin embargo, a partir de 2017, se priorizó el diseño de estrategias formativas para introducir las propuestas de AET a maestros que actúan en el $4^{\circ}$ año. De este modo, los pilotos subsecuentes permitieron avanzar simultáneamente en la comprensión del funcionamiento de las actividades en sala, tanto desde el punto de vista de los alumnos como del de los profesores. 


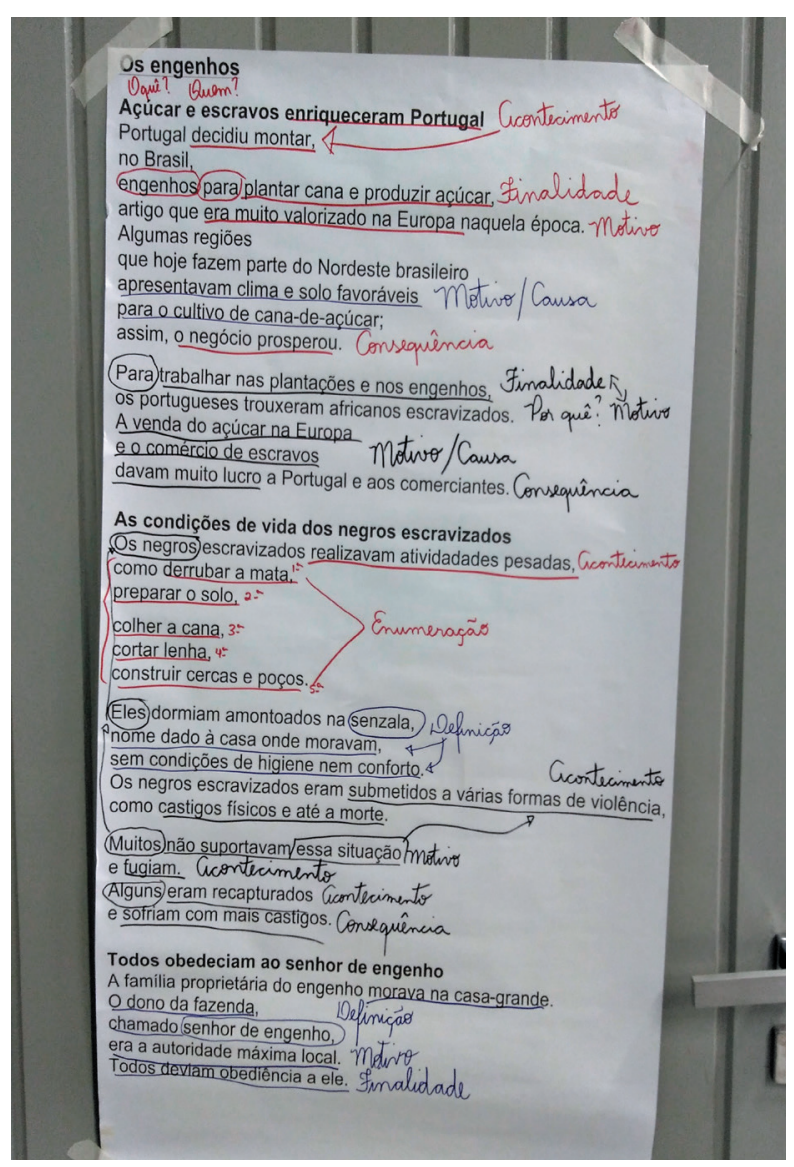

Figura 1. Ejemplo del resultado de la actividad de anotación del contenido y estructura informativa del texto reformateado.

Fuente: Documentación fotográfica. Póster anotado, profesora B, secuencia 4.

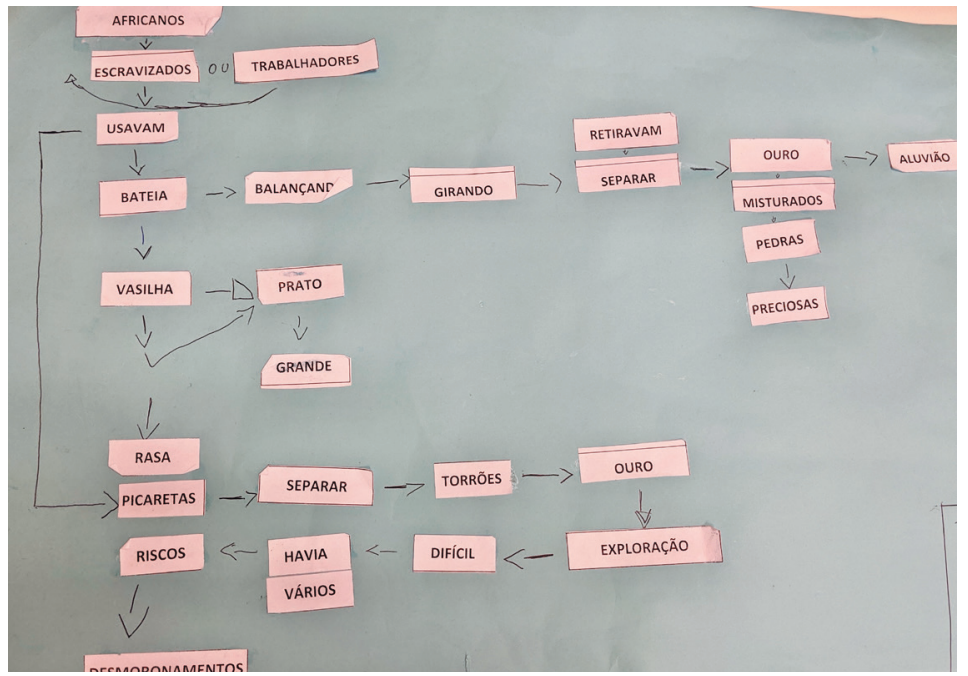

Figura 2. Ejemplo de actividades de estudio del vocabulario del texto.

Fuente: Documentación fotográfica, esquemas realizados en grupo, grupo C, secuencia 3. 
La formación profesional de los maestros es una pieza clave para promover nuevas prácticas en el aula. Las investigaciones sobre las creencias de los maestros acerca de la lectura de textos señalan que, al pensar sobre la enseñanza de la lectura de textos disciplinares, los maestros no acostumbran a atender a las estructuras textuales y discursivas propias de cada área del conocimiento como un objeto de aprendizaje (Maggioni, Fox \& Alexander, 2015). La capacidad de reconocer y proyectar nuevos modos de enseñar, en consonancia con principios pedagógicos específicos, ha sido objeto de estudio en los últimos 30 años. Se inició con la conceptualización del conocimiento pedagógico del contenido (Shulman, 1986, 1987) como un elemento necesario para que los profesores se transformen en profesionales que actúan de manera crítica y reflexiva. En esta perspectiva, se diferencia el conocimiento general del contenido de un campo disciplinar del contenido a ser enseñado, considerando aspectos estructurantes y aspectos periféricos de la disciplina, anticipando estrategias de trabajo con los alumnos, bien como respuestas e incomprensiones posibles, entre otros. AET propone que el conocimiento pedagógico del contenido necesario para enseñar historia utilizando textos escritos contemple también una comprensión de las características del lenguaje utilizado en esos textos.

\section{Objetivo y preguntas de la investigación}

Este trabajo se suma a otros estudios piloto realizados con el objetivo de evaluar el diseño de las propuestas didácticas de AET (Sepúlveda, 2015, 2017a; Sepúlveda, Bisognin \& Paulet, 2017) y desarrollar un programa de formación docente (Laboratório de educação, 2021). En este estudio en particular, nos propusimos identificar el modo en que dos profesoras de $4^{\circ}$ ańo de educación primaria, que implementaron durante cuatro meses las propuestas de AET, fueron modificando sus prácticas de lectura de textos escolares en el área de Historia. Nos preguntamos cuáles fueron los cambios introducidos y si modificaron sus prácticas de la misma manera.

\section{Metodología}

\section{Participantes}

El estudio piloto fue implementado en dos cursos de $4^{\circ}$ año de primaria, de acuerdo con procedimientos éticos vigentes en Brasil durante la recolección de datos. Cabe destacar que, a pesar de su ubicación en un distrito de clase media-alta de la ciudad, muchos de los alumnos que frecuentan la escuela donde se llevó a cabo la intervención viven en un asentamiento informal localizado en sus proximidades. Por lo tanto, son alumnos que presentan niveles diversos de vulnerabilidad social.

Cada clase estaba conformada por una maestra y 21 alumnos. Las maestras participantes, denominadas profesora A y profesora B, son educadoras con una carrera efectiva en la red municipal de la ciudad de São Paulo, poseen formación universitaria hasta el nivel de posgrado ${ }^{4}$ y cuentan con siete y 12 años de experiencia de trabajo en sala, respectivamente. Ambas se dispusieron libremente a participar en la intervención formativa enfocada en las prácticas instruccionales de lectura de textos escolares en la clase de Historia.

\section{Procedimiento}

El procedimiento de levantamiento de datos ocurrió en tres etapas. La primera tuvo el objetivo de caracterizar la práctica de las profesoras participantes y su perfil pedagógico antes de usar las propuestas de AET. Para esto, fue realizada una entrevista y, posteriormente, la observación directa de una clase de Historia en la que las dos profesoras trabajaron sobre el mismo texto.

4. En Brasil hay una amplia oferta de cursos universitarios privados de baja calidad, muchos de ellos a distancia, que tienen un peso significativo en la progresión de la carrera y remuneración del docente (Gatti, Barretto, Andre \& Almeida, 2019). 
La siguiente etapa consistió en promover y documentar el uso de las propuestas de AET a lo largo de dos bimestres $^{5}$. Para esto, se ofrecieron dos sesiones formativas introductorias y reuniones semanales de planificación. En dichas reuniones, fueron exploradas las características de los textos presentes en el libro didáctico ${ }^{6}$ adoptado por la escuela como parte del Programa Nacional del Libro Didáctico y se discutieron las secuencias de actividades de enseñanza y aprendizaje que se pondrían en práctica con los alumnos. A lo largo de la intervención las profesoras trabajaron sobre los mismos textos y con la misma planificación de actividades. Las propuestas de lectura y análisis fueron elaboradas a partir de textos ya incluidos en la programación bimestral de las profesoras, teniendo en consideración la previsión curricular de la escuela.

Todas las actividades realizadas durante la intervención fueron observadas directamente por las investigadoras dos veces por semana. En total, fueron registradas en audio o video 18 sesiones de 45 a 60 minutos. Los datos fueron transcritos y codificados con el programa de análisis de datos Atlas/ti, versión 5.5. (Atlas.ti Scientific Software Development GMBH, 2002/2011). Además, se compilaron los materiales educativos producidos por los alumnos individualmente, en pequeños grupos y colectivamente junto a la profesora.

Finalmente, el cierre del trabajo de campo incluyó una entrevista final con cada profesora para capturar su valoración general de la intervención.

\section{Análisis}

En este estudio nos centramos en los datos procedentes del registro de las actividades y discurso educativo utilizado en las clases de Historia en torno a la lectura de textos del libro didáctico antes y después de conocer las propuestas de AET. Los análisis estuvieron orientados, por una parte, a caracterizar la práctica inicial de las profesoras y, por otra, a identificar los cambios introducidos en sus prácticas educativas a partir de la incorporación de las propuestas de AET.

Con estos objetivos, realizamos los siguientes análisis:

- Análisis del contenido de la entrevista inicial. Identificamos las actividades relatadas por las profesoras para describir sus clases típicas del área de Historia.

- Identificación de los segmentos de actividad y el uso del tiempo. A partir de la propuesta de Coll, Onrubia y Mauri (2008) de análisis de las formas de organización de la actividad educativa entre profesores y alumnos en torno de una tarea o contenido de aprendizaje, distinguimos las acciones realizadas durante las clases observadas y el tiempo dedicado a cada una de ellas. Así, segmentamos y codificamos el modo en que fue cambiando la actividad de profesora y alumnos en torno a la lectura y comentario de un texto durante la sesión de clase. Este proceso de codificación no partió de unas categorías predeterminadas, sino que diferenció y denominó los cambios en la actividad conjunta de los participantes, por ejemplo: observación y comentario de las imágenes del texto, lectura en voz alta, síntesis de conocimientos previos, trabajo en pequeńos grupos.

5. Para conocer en detalle los materiales del programa, consultar Laboratório de educação, 2021.

6. En este artículo utilizamos el término libro didáctico para referirnos a los libros de texto o manuales escolares distribuidos gratuitamente por el Ministerio de Educación de Brasil como instrumentos para apoyar los procesos de enseñanza y aprendizaje de las diferentes áreas del currículum.

7. El Programa Nacional del Libro Didáctico (PNLD) distribuye anualmente libros didácticos a todos los alumnos matriculados en escuelas públicas de Brasil. Los directores y coordinadores pedagógicos de cada unidad escolar organizan el proceso de selección de los libros a partir de una selección previamente realizada por una comisión técnica del Ministerio de Educación. Solo en 2020, 32 millones de estudiantes fueron beneficiarios de esta política educativa (Fundo Nacional de Desenvolvimento da Educação, 2020). 
- Comparación de la actividad discursiva centrada en el comentario del contenido informativo de los textos antes y durante la intervención. Para realizar este análisis, se prosiguió de la siguiente manera. En primer lugar, se delimitó la unidad de análisis, los enunciados, "entendidos como el producto concreto y tangible de un proceso de enunciación realizado por un enunciador y destinado a un enunciatario" (Calsamiglia \& Tusón, 2007, p. 3); en nuestro caso, los actos de habla realizados por la profesora dirigidos a sus alumnos y de los alumnos dirigidos a su profesora. En segundo lugar, a partir de nuestra experiencia previa en el análisis del discurso educativo en torno a la lectura de textos (Sepúlveda, 2012, 2017b; Teberosky et al., 2006), codificamos los enunciados realizados sobre el texto leído, excluyendo del análisis aquellos enunciados sobre los procesos de enseńanza (vamos a abrir el libro en la página 25) o de gestión del comportamiento y participación en la clase (profesora, ya es hora del recreo). Aquellos enunciados sobre el texto leído fueron codificados según el tipo de acto discursivo realizado, por ejemplo: citar, parafrasear, explicar, definir, preguntar, responder, y según su contenido informativo, tanto conceptual (participantes, acontecimientos, lugar, tiempo) como discursivo (referencia anafórica, conector discursivo, palabra sinónima). Así, por ejemplo, codificamos la actividad discursiva de las profesoras sobre el texto como: paráfrasis de los acontecimientos (Entonces, está diciendo que las tierras estaban ocupadas por los indigenas cariri), pregunta sobre los participantes (¿Quién vivía en los quilombos?), pregunta sobre los acontecimientos (¿Qué sucedió?), define término (confederación es un grupo de personas), explícita la referencia anafórica ("ellos" ¿Quiénes? Los colonizadores), y de los alumnos como: responden identificando los participantes (los indígenas cariri), responden identificando la relación de causalidad (los colonos querían más tierras), responden identificando el tiempo de los acontecimientos (antes de la llegada de los portugueses), preguntan el significado de un término (¿Qué es "sesmaria", profesora?), entre otros. En tercer lugar, agrupamos los códigos que hacían referencia al contenido informativo de los textos, tanto en el caso de las profesoras como en el de los alumnos y, de esta manera, cuantificamos los enunciados realizados en la clase con la intención de reproducir o comentar el contenido del texto leído, excluyendo aquellos de carácter metadiscursivo, como los relacionados con las referencias anafóricas (esta es una palabra que remite a otra palabra mencionada antes), el tipo de conectores ("porque", tenemos que estar muy atentos cuando encontremos un "porque", quiere decir que nos van a explicar la causa de lo que pasó), secuencias textuales (en este párrafo están dando ejemplos). Finalmente, seleccionamos para la comparación la clase registrada antes de la intervención y una correspondiente a un momento intermedio, porque fueron clases de estructura, contenido y duración equivalente. Es decir, en las clases comparadas las profesoras leyeron los mismos textos y realizaron las mismas actividades de estudio en tiempos aproximados. Los datos de las sesiones comparadas fueron codificados por las autoras de forma independiente, el grado de acuerdo inter observadores osciló entre $84 \%$ y $99 \%$.

\section{Resultados}

Teniendo en cuenta los objetivos del estudio, en primer lugar, presentamos la caracterización de la práctica educativa de las profesoras antes de la intervención. En este apartado, identificamos en qué medida las prácticas habituales referidas por las profesoras durante la entrevista inicial, y exhibidas en clase, difieren o se asemejan entre sí, y también el modo en que dialogaban con las propuestas de AET. En segundo lugar, presentamos los cambios identificados en las prácticas a lo largo de la intervención. En este segundo apartado identificamos cambios en el uso del tiempo y en la actividad discursiva de las profesoras durante la lectura en voz alta y el estudio de los textos. Finalmente, presentamos los cambios observados en la frecuencia de la actividad discursiva centrada en el comentario del contenido informativo de los textos.

\section{Caracterización inicial de la práctica educativa de las docentes}

Durante la entrevista inicial, las profesoras caracterizaron sus prácticas usuales de enseñanza en la disciplina de Historia como basadas en la lectura de textos y en el desarrollo de actividades propuestas en los libros didácticos recibidos gratuitamente del Ministerio de Educación por medio del Programa Nacional del Libro Didáctico. La profesora B señaló: "El material base es el libro de texto; la planificación se hace a partir de esos contenidos. Usamos los textos en clase, las actividades que propone el libro". 
Al detallar en qué consistía una secuencia típica de actividades de sus clases de Historia, ambas maestras citaron:

- Lectura en voz alta dividida por turnos entre los estudiantes.

- Desarrollo de las actividades propuestas en el libro didáctico y registro individual de las respuestas en el cuaderno.

- Corrección de las actividades en la pizarra dirigida por la profesora.

- Copia individual de las respuestas corregidas en el cuaderno por parte de los estudiantes.

Las principales diferencias mencionadas fueron la realización de una lectura en voz alta por parte de la profesora A y la explicación y comentario de los contenidos del texto en el caso de la profesora B.

La observación directa permitió confirmar esta descripción y reconocer indicios de diferentes perfiles de actuación pedagógica de cada una de las participantes. El cuadro 1 enumera las actividades observadas y el tiempo dedicado a cada una de ellas.

\section{Cuadro 1}

Actividades observadas en una clase típica de Historia antes de la intervención.

Profesora A

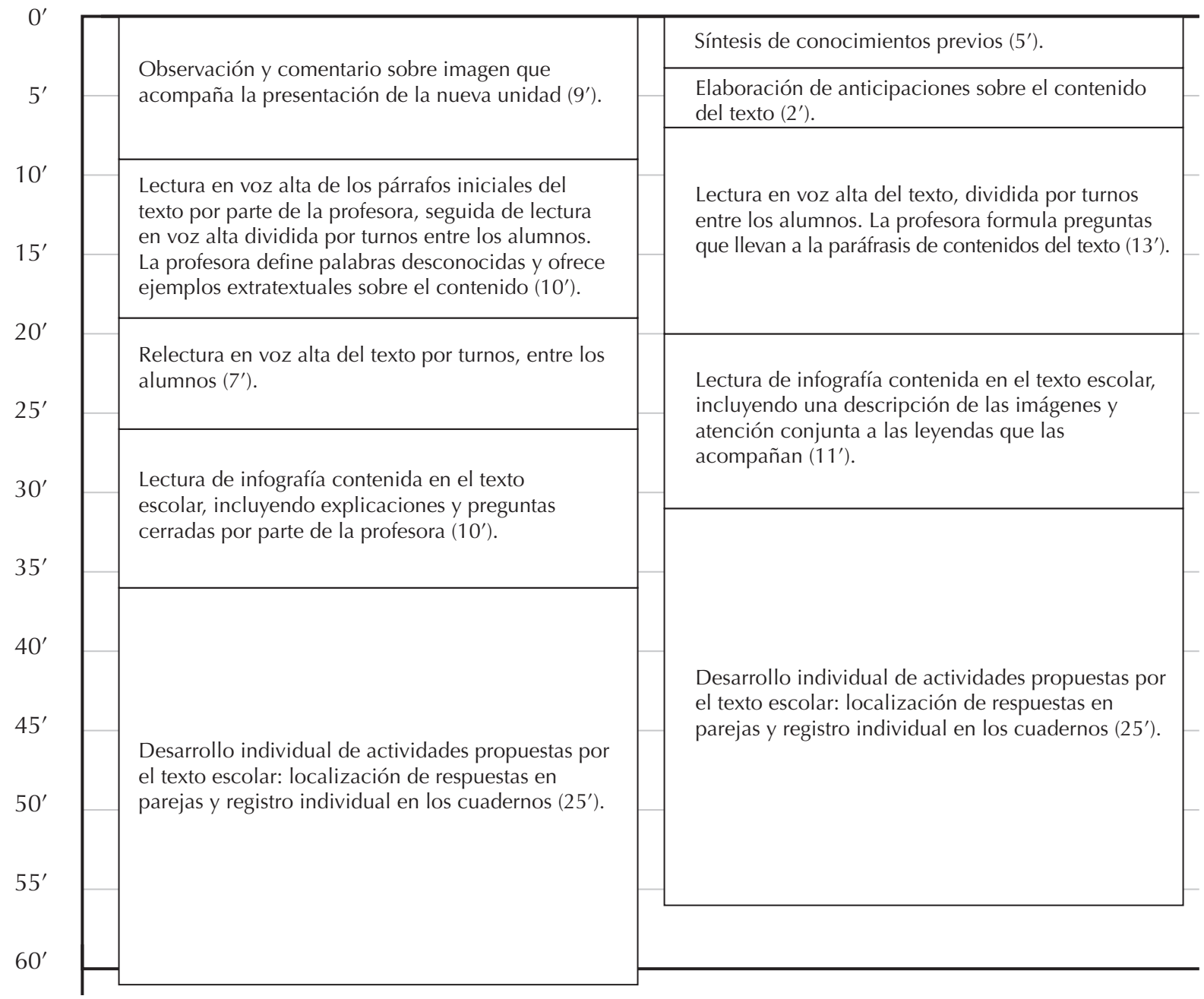

Fuente: Elaboración propia. 
De manera general, constatamos que las maestras desarrollaron los mismos tipos de actividades por una duración equivalente: los alumnos leyeron el texto en voz alta por turnos, posteriormente leyeron y comentaron una infografía contenida en el libro didáctico, y respondieron a las preguntas que acompañaban al texto en sus respectivos cuadernos. Ninguna profesora realizó la lectura en voz alta del texto en su totalidad.

Sin embargo, también se evidenciaron algunas diferencias. La profesora B inició la clase con un momento dedicado a la recuperación de conocimientos previos y a la formulación de anticipaciones sobre el contenido que sería abordado en el nuevo texto a partir de su título. Además, después que los alumnos leyeron el texto en voz alta, la maestra estimuló la paráfrasis de su contenido mediante preguntas, como vemos en el siguiente ejemplo:

Profesora: Y ¿por qué? ¿Por qué no plantaban [caña de azúcar] allá?

Alumno: Porque allá hacía mucho frío...

Profesora: El clima no era bueno y el suelo tampoco.

Los contrastes entre las participantes no se restringieron a la inclusión de tales actividades en momentos específicos antes y durante la lectura, sino que se revelaron también en los modos de interacción sostenida con los niños: solo en el caso de la profesora B se registraron intercambios en que la maestra amplió o comentó las respuestas de los alumnos, ofreciendo explicaciones complementarias sobre los hechos narrados. En el aula de la profesora A, las preguntas que dirigió a los alumnos se limitaron a indagar sobre el significado de palabras desconocidas, y no fueron observadas instancias de comentario o reformulación de las contribuciones de los niños. Estas observaciones revelaron el perfil pedagógico más transmisivo de la profesora A y más interactivo de la profesora $\mathrm{B}$.

\section{Cambios en las intervenciones didácticas durante la lectura y estudio de los textos}

La tabla 1 compara, en tres momentos de la intervención, el tiempo dedicado por cada profesora al desarrollo de las actividades propuestas por AET para la lectura en voz alta y el estudio del texto. Utilizamos los datos correspondientes a la primera secuencia de actividades planificada con las investigadoras (S1), una realizada en la mitad de la intervención (S4) y la secuencia final de actividades (S7). El tiempo dedicado a lectura en voz alta registra el lapso transcurrido entre la lectura de la primera y la última línea del texto. En el caso de la actividad de estudio del texto, medimos el tiempo de la sesión dedicado a la relectura y comentario de aspectos salientes del contenido informativo y estructura discursiva de los textos.

Tabla 1

Comparación del uso del tiempo, en minutos, al inicio, medio y final de la intervención.

\begin{tabular}{ccccccc}
\multirow{2}{*}{ Actividad } & \multicolumn{3}{c}{ Profesora A } & \multicolumn{3}{c}{ Profesora B } \\
\cline { 2 - 7 } & S1 & S4 & S7 & S1 & S4 & S7 \\
\hline Leer y comentar el texto & $23^{\prime}$ & $30^{\prime}$ & $2^{\prime}$ & $6^{\prime}$ & $9^{\prime}$ & $3^{\prime}$ \\
\hline Estudiar el texto & $0^{\prime}$ & $30^{\prime}$ & $15^{\prime}$ & $17^{\prime}$ & $32^{\prime}$ & $33^{\prime}$ \\
\hline
\end{tabular}

Fuente: Elaboración propia.

Los datos relativos al uso del tiempo (ver tabla 1) muestran diferencias en el modo en que se fueron consolidando, a lo largo de la intervención, cambios en las prácticas pedagógicas de cada profesora. En el caso de la profesora A, registramos que en S1 leyó, comentó y analizó el texto de forma simultánea, es decir, no diferenció entre la actividad de lectura inicial del texto y su estudio. Por esto, no contabilizamos un tiempo dedicado exclusivamente 
a la relectura y análisis del texto. En S4, aunque todavía no diferenciaba entre una lectura inicial del texto y el comentario en mayor profundidad, sí dedicó tiempo específico a releer y anotar el texto. Finalmente, en S7, después de reflexiones sucesivas durante las reuniones de planificación de actividades, la profesora optó por realizar una primera lectura expresiva continua del texto, lo que dio lugar a que tuviera una primera conversación general con los alumnos sobre los temas del texto y justificara la actividad de relectura y análisis.

A diferencia de la profesora $\mathrm{A}$, los datos correspondientes al uso del tiempo por parte de la profesora $\mathrm{B}$ muestran que incorporó desde un inicio la lectura en voz alta de enunciados o párrafos completos, antes de formular preguntas que llevaran a los estudiantes a identificar el tema o las ideas principales del texto. Esta diferencia se manifiesta en la duración de los segmentos de actividad dedicados a la lectura en voz alta: 6' (S1), 9' (S4) y 3' (S7). En cuanto al tiempo dedicado a la relectura y análisis del texto, observamos que entre S1(17') y S7 (33') pasó a dedicar el doble del tiempo al desarrollo de esta actividad.

El análisis del discurso educativo también nos permitió identificar los cambios que fueron introduciendo las profesoras a lo largo de la intervención. En el caso de la profesora A, pasó en S1 de realizar una primera lectura del texto interrumpida para formular preguntas orientadas a la recuperación de la información leída y definición de palabras desconocidas a incorporar en, S4 y S7, comentarios y preguntas orientados al análisis del contenido informativo propio de los textos escolares de Historia: agentes, causas, lugares, acontecimientos, como en los ejemplos a continuación:

"[...] Entonces comenzaron a rebelarse, ¿̇de qué forma? Quemando lo que sus dueños los mandaban a plantar y huían" (recuperación de información leída, profesora A, S1).

"¿Qué es cautiverio? [...] Aquí estamos hablando de cautiverio como una situación, un modo de vida..." (definición de cautiverio, profesora A, S1).

“QQuién formaba parte de las cámaras municipales? ¿Por qué? (pregunta orientada a la recuperación de agentes y a la paráfrasis de una causa mencionada en fragmento leído, profesora A, S7).

También destacamos que de forma sistemática pasó a explicitar la función de diversos mecanismos de referencia utilizados en los textos, como se observa en los siguientes ejemplos:

“[...] En estos lugares 'en estos lugares', ¿¿de qué lugares estoy hablando?” (pregunta orientada a la identificación del lugar referido con el demostrativo estos, profesora A, S1).

"Ellos podian distribuir el derecho de uso de las tierras... 'ellos', ¿quiénes?" (pregunta orientada a la restitución de agentes referidos con el pronombre ellos, profesora A, S4).

En el caso de la profesora B, en la caracterización inicial ya habíamos registrado que promovía, en sus interacciones en torno al texto, la actividad de formular preguntas para recuperar y parafrasear el contenido del texto. Con AET, logró hacer un trabajo más intencionado de recuperar y comentar la participación de los alumnos. Y así como la profesora A pasó a incluir explícitamente la atención a las unidades informativas propias de los textos de Historia, bien como a los recursos utilizados para verificar la comprensión de las referencias anafóricas. Por ejemplo, la maestra hizo preguntas orientadas a la localización temporal de acontecimientos específicos citados en el texto, como se observa en el siguiente diálogo ocurrido durante la S7:

Profesora: La lucha duró mucho tiempo, hasta que, en 1713, tropas paulistas derrotaron a la confederación de los Cariri... ¿¿de qué nos está hablando este pedazo?

Alumno: De la lucha. 
Profesora: Está hablando de la lucha, de la disputa, ¿no? Entonces, esta lucha [...] duró mucho tiempo. ¿Cuándo acabó?

Alumno: 1713.

Profesora: Entonces, ¿Esto [señalando en 1713] qué es?

$[\ldots]$

Profesora: Es cuando, cuando.

También fue destacable que, usando las propuestas de AET, la profesora pasó a integrar de forma adecuada el contraste entre las ideas discutidas en la preparación para la lectura del texto y la comprensión obtenida después de ella. Como se observa en el siguiente ejemplo, en S7 la maestra recuperó las hipótesis registradas en la pizarra antes de la lectura del texto y aprovechó las dudas de los niños sobre qué personajes históricos estuvieron involucrados en los conflictos sobre la posesión de la tierra para justificar su relectura.

Profesora: ¿Qué vamos a hacer ahora? ¿Lo que ustedes habían dicho inicialmente tenía sentido o no? Vamos a ver. ¿¿Se está hablando de las tierras de Brasil? [...] Y ¿̨entre quiénes se daban los conflictos sobre los que el texto habla?

Alumno: Indígenas y portugueses.

Profesora: Los indios con los portugueses, y los Cariri. Sí, ¿¿no? Y ¿̇os holandeses con los portugueses?

Alumno: No.

Profesora: En este episodio, no. $Y_{i}$ los vaqueros con los indios? [...] ¿Los vaqueros entraron en algún conflicto?

Alumno: Sí.

Alumno: No.

Profesora: Vamos a ver de nuevo, entonces. Voy a colocar el texto en la pizarra... [para la relectura].

Estos resultados muestran que las maestras fueron incorporando gradualmente la actividad de lectura en voz alta expresiva dirigida a los alumnos, diferenciándola de las lecturas analíticas posteriores. También muestra la gradual incorporación de la participación activa de los estudiantes como el hilo conductor entre ambas actividades.

\section{Cambios en la frecuencia de la actividad discursiva centrada en la referencia al contenido informativo del texto}

Uno de los principales cambios que se produjeron en las prácticas educativas realizadas por las profesoras en torno de la lectura y comentario de los textos escolares consistió en aumentar las oportunidades de los niños para atender, recuperar, comentar y analizar el contenido informativo del texto. La categorización exhaustiva del discurso educativo registrado en cada una de las sesiones lo puso en evidencia. 
El gráfico 1 compara la actividad discursiva dedicada por cada profesora y sus alumnos para parafrasear el contenido del texto antes del inicio de la intervención (S0) y en un momento intermedio (S4), en el que las dos profesoras realizaron los procedimientos de relectura y anotación planeados en una misma sesión, sobre un mismo texto y en tiempos equivalentes: 30 ' profesora A y 32' profesora B.

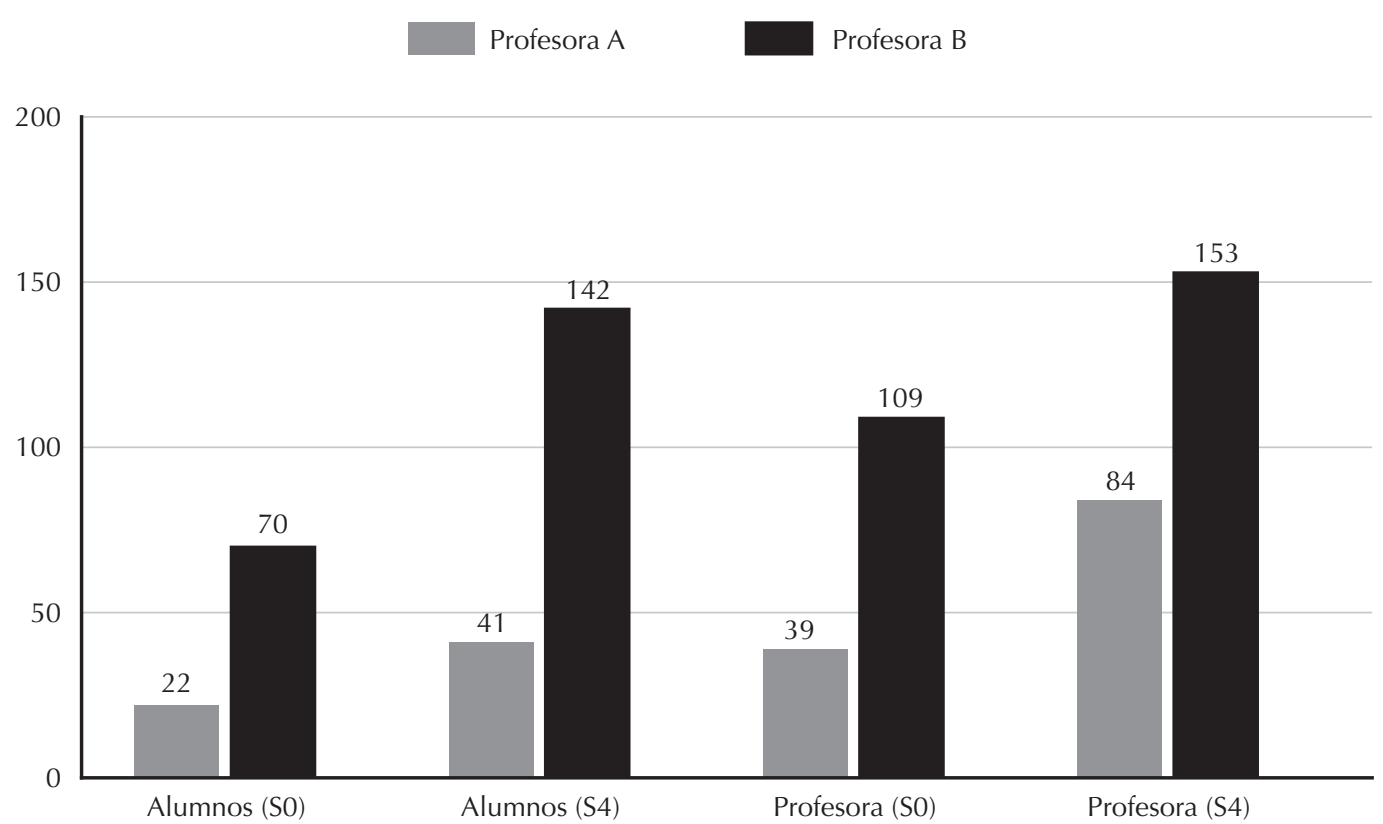

Gráfico 1. Actividad discursiva dedicada al comentario del contenido informativo del texto, medida en número de enunciados por clase.

Fuente: Elaboración propia.

El gráfico 1 nos permite observar que, en comparación con la actividad realizada antes de AET (S0), las dos profesoras aumentaron las oportunidades ofrecidas a sus alumnos para escuchar y realizar comentarios sobre el contenido del texto. En el caso de la profesora A, el número de enunciados centrados en la paráfrasis del contenido del texto dobló en comparación con la actividad registrada antes de la intervención. Sin embargo, se mantuvo la distancia registrada entre la actividad discursiva de la profesora y la de los alumnos. Este resultado indica que las propuestas de AET, a pesar de no haber modificado el estilo de interacción de la profesora A, lograron efectuar cambios en su práctica que dieron mayor relieve al contenido disciplinar estudiado.

En el caso de la clase de la profesora B, la participación de los alumnos aumentó de forma expresiva y casi llegó a equiparar el número de intervenciones de la docente. Este dato sugiere que el estilo pedagógico más interactivo registrado en la caracterización inicial de la práctica de la profesora $\mathrm{B}$ se acentuó con el trabajo realizado a partir de las secuencias didácticas propuestas por AET.

\section{Discusión y conclusiones}

En esta investigación nos preguntamos por los cambios en las prácticas de lectura de textos escolares realizadas por dos profesoras de $4^{\circ}$ año de educación primaria a partir del uso de las propuestas didácticas de AET. En la caracterización inicial de la práctica educativa de las participantes registramos que, antes de introducir las 
propuestas de AET, la lectura de textos escolares en la disciplina de Historia tenía un carácter mecánico y superficial. Las prácticas de las profesoras consistían en pedir a los alumnos leer el texto en voz alta por turnos, escuchar explicaciones breves de su parte y realizar actividades de trabajo individual o copia en el cuaderno desarrolladas en una única sesión de clase de una hora aproximadamente.

Con base en las propuestas de AET y en el trabajo reflexivo realizado por las profesoras a través de la intervención, los alumnos pasaron a tener la oportunidad de escuchar los textos leídos en voz alta por sus profesoras de forma expresiva e intencionada, enfatizando el contenido informativo del texto y sus recursos discursivos. Durante varias sesiones de clase pudieron observar las acciones propias de la lectura analítica de textos, participar en la identificación de sus principales unidades de información, advertir los mecanismos para mantener la referencia y la función de los marcadores discursivos, y así avanzar en el conocimiento del lenguaje de los textos y la comprensión de los contenidos trabajados.

Investigaciones previas en educación secundaria ya habían reportado los beneficios del análisis lingüístico de los textos para el aprendizaje de contenidos curriculares. En el área de historia los trabajos de Mary Schleppregell (Schleppegrell \& Achugar, 2003; Schleppegrell et al., 2004) y de Gilian Moss (Moss, 2009) han destacado la necesidad de formar a los maestros en estrategias de análisis del lenguaje de los textos como medio para mejorar los niveles de comprensión de sus alumnos y también como práctica docente para promover el desarrollo de habilidades de lenguaje académico. Algunos estudios de caso (Moss, 2009; Schleppegrell \& Oteíza, 2006) relatan que, durante las conversaciones de análisis del lenguaje de los textos, profesores y alumnos consiguen profundizar en los contenidos históricos estudiados y por esto obtienen mejores resultados de aprendizaje.

En educación primaria, el trabajo de Teberosky y sus colegas (2013) exploró el uso didáctico de diferentes estrategias y materiales para ayudar en el proceso de lectura, estudio y representación multimodal de textos de historia. Sus análisis de representaciones multimodales producidas por alumnos de $6^{\circ}$ año muestran que recurrir a estrategias como la visualización del texto reformateado per cola et commata, la lectura en voz alta expresiva de la profesora dirigida a los alumnos, el análisis y anotación del lenguaje y unidades informativas clave del texto, y la manipulación del léxico del texto son prácticas docentes que pueden beneficiar la comprensión de los alumnos de contenidos disciplinares específicos. En este estudio, aunque no se analizó cada uno de estos tipos de prácticas, estas sí caracterizaron las condiciones educativas de los resultados obtenidos.

Por otra parte, en nuestro trabajo observamos que, si bien dirigir la atención de profesores y alumnos hacia los usos académicos del lenguaje en los textos aumenta las oportunidades de que los estudiantes reciban ayuda para comprender su contenido informativo, el tipo y la calidad de la ayuda educativa varía de forma importante en función del estilo interactivo y pedagógico de las maestras. Esta puede ser una contribución que introduce matices a los estudios anteriores.

Si bien no fue una condición buscada por la investigación, las maestras que participaron en este estudio representaron estilos pedagógicos diferenciados. La profesora $\mathrm{A}$, con una intervención educativa más directiva, y la profesora $\mathrm{B}$, con una intervención educativa más centrada en los alumnos, interactiva y constructiva. De este modo, pese a que registramos cambios en las intervenciones educativas de las dos maestras, el análisis de la actividad discursiva centrada en el comentario del contenido informativo del texto puso en evidencia que, desarrollando la misma actividad y durante un lapso equivalente, la profesora B ofreció más oportunidades a sus alumnos para atender y referir el contenido textual. Investigaciones centradas en el estudio de las relaciones entre el discurso educativo y los procesos de enseñanza y aprendizaje en el salón de clases ya han destacado el rol del estilo interactivo de los profesores como un factor clave en la calidad de las oportunidades de aprendizaje ofrecidas a los alumnos (Mercer, 2003; Sepúlveda, 2017b). 
En general, estos hallazgos resaltan los desafíos que enfrentan los programas que buscan contribuir a la formación continua como AET. Aunque el material y la metodología propuestos cumplen una función importante, el alcance de los resultados depende de otras variables, como las concepciones docentes sobre los procesos de enseñanza y de aprendizaje y su capacidad de organizar la gestión pedagógica del aula. A esto se suma la necesidad de articular, en la práctica, el conocimiento sobre el lenguaje como objeto de enseñanza con el conocimiento pedagógico específico de este tipo de contenido (Shulman \& Shulman, 2016).

Por esta razón, la principal contribución de este estudio consistió en ofrecer las bases para diseñar un proceso formativo que adopta una estructura cíclica basada en estrategias de observación, modelaje, planificación conjunta y análisis de registros de implementación que favorecen el desarrollo de conocimientos pedagógicos en contexto (https://aprenderaestudartextos.org.br/). Los ciclos de formación permiten que los maestros reflexionen sobre las oportunidades de aprendizaje posibilitadas por diferentes modos de poner en práctica un conjunto de propuestas como las de AET y experimenten, con apoyo de un formador, la incorporación de modificaciones intencionales a lo largo de un período más extenso que el de esta investigación.

En conclusión, los docentes tienen la responsabilidad de promover las condiciones para que cada uno de sus alumnos desarrolle la capacidad de leer y comprender textos, es decir, de construir significados a partir de los textos para acceder al conocimiento producido socialmente. El abordaje de AET se suma a una extensa tradición de propuestas de intervenciones educativas que promueven que la enseñanza de disciplinas académicas supone la enseñanza del lenguaje de esas disciplinas. Reconocer y enseñar de forma intencionada lo común y lo específico al lenguaje académico de los diferentes campos disciplinares es una tarea en la que vale la pena continuar trabajando. Dada la diversidad de perfiles y experiencias representadas en el cuerpo docente de las escuelas públicas y privadas, la apropiación de dichas prácticas depende de su inserción consistente en los programas de formación ofrecidos por los sistemas educativos.

Agradecimientos: Agradecemos especialmente a Andrea Guida Bisognin por la coordinación general del proyecto entre los años 2012 - 2018, y a los apoyadores institucionales de la organización no gubernamental Laboratório de educação.

El artículo original fue recibido el 15 de enero de 2021

El artículo revisado fue recibido el 5 de julio de 2021

El artículo fue aceptado el 13 de julio de 2021

\section{Referencias}

Aisenberg, B. (2007). Ayudar a leer "en sociales". Quehacer educativo, 83, 24-27.

Atlas.ti Scientific Software Development GMBH [Programa informático]. (2002/2011). Recuperado de Atlas.ti.

Barletta, N. \& Mizuno, J. (2005). Una propuesta para el manejo del lenguaje del texto de ciencias naturales. Zona próxima: revista del Instituto de Estudios Superiores en Educación, (6), 32-47.

Biemiller, A. (1999). Language and reading success. Newton Upper Falls, MA: Brookline.

Blanche-Benveniste, C. (2008). Les unités de la langue écrite et de la langue parlée. Cahiers de l'Université de Perpignan, (37), 192-217.

Bortoni-Ricardo, S.M. (2008). Leitura de livros didáticos: Uma situação negligenciada. Linguagem em (Dis)curso, 8(3), 613-640. https://doi.org/10.1590/S1518-76322008000300010

Calsamiglia, H. \& Tusón, A. (2007). Las cosas del decir. Manual de análisis del discurso (2a ed.). Barcelona, España: Ariel. 
Chamorro, D., Barletta, N. \& Mizuno, J. (2013). El lenguaje para enseñar y aprender las Ciencias Naturales: un caso de oportunidades perdidas para la formación ciudadana. Revista Signos. Estudios de Lingüística, 46(81), 3-28. https://doi.org/10.4067/S0718-09342013000100001

Coffin, C. (1997). Constructing and giving value to the past: An investigation into secondary school history. En F. Christie \& J. R. Martin (Eds.), Genre and institutions: Social processes in the workplace and school (pp. 196-230). Londres, Inglaterra: Cassell.

Coffin, C. (2006). Historical discourse. The language of time, cause and evaluation. Londres, Inglaterra: Continuum.

Coll, C., Onrubia, J. \& Mauri, T. (2008). Ayudar a aprender en contextos educativos: el ejercicio de la influencia educativa y el análisis de la enseñanza. Revista de Educación, 346, 33-70.

Fang, Z. \& Schleppegrell, M. J. (2008). Reading in Secondary Content Areas. A language-based pedagogy. Ann Arbor, MI: The University of Michigan Press.

Fang, Z., Schleppegrell, M. J. \& Cox, B.E. (2006). Understanding the language demands of schooling: Nouns in academic registers. Journal of Literacy Research, 38(3), 247-273. https://doi.org/10.1207/s15548430jlr3803_1

Fundo Nacional de Desenvolvimento da Educação (2020). Dados estatísticos. Programas do livro. Recuperado de https://www.fnde.gov.br/index.php/programas/programas-do-livro/pnld/dados-estatisticos

Gatti, B. A., Barretto, E. S. de S., Andre, M. E. D. A. de \& Almeida, P. C. A. de. (2019). Professores do Brasil: novos cenários de formação. Brasilia, Brasil: Unesco.

Halliday, M. A. K. (1998). Language and knowledge: The 'Unpacking' of text. En D. Allison, L. Wee, B. Zhiming \& S. Abraham. (Eds.), Text in education and society (pp. 157-178). Singapur: Singapore University Press.

Halliday, M. A. K. \& Martin, J. R. (1993). Writing Science: Literacy and discursive power. Pittsburgh, PA: University of Pittsburgh Press.

Laboratório de educação. (2019). Aprender a estudar textos. História no $4^{\circ}$ e no $5^{\circ}$ ano do ensino fundamental: caderno de referência. São Paulo, Brasil: Laboratório de educação.

Laboratório de educação. (2021). Aprender a estudar textos. https://aprenderaestudartextos.org.br/

Maggioni, L., Fox, E. \& Alexander, P. A. (2015). Beliefs about reading, text, and learning from text. En H. Fives and M. G. Gill (Eds.), International Handbook of Research on Teachers' Beliefs (pp. 353-382). Nueva York, NY: Routledge.

Martin, J. R. \& Rose, D. (2008). Genre relations. Mapping culture. Londres, Inglaterra: Equinox Publishing Ltd.

Meneses, A., Uccelli, P., Santelices, M. V., Ruiz, M., Acevedo, D. \& Figueroa, J. (2018). Academic language as a predictor of reading comprehension in monolingual Spanish- speaking readers: Evidence from Chilean early adolescents. Reading Research Quarterly, 53(2), 223-247. https://doi.org/10.1002/rrq.192

Mercer, N. (2003). Helping children to talk and think together more effectively. Polifonia, 7(07), 1-26.

Moss, G. (2000). The language of school textbooks and the ideology of science. Zona Próxima, (1), 44-55.

Moss, G. (2006). Textbook language, teacher mediation, classroom interaction and learning process: The case of natural and social science textbooks in Barranquilla, Colombia. En B. Leial \& T. Berber Sardinha (Eds.), Proceedings of the 33rd International Systemic Functional Congress (pp. 879-894). São Paulo, Brasil: PUCSP.

Moss, G. (2009). Los textos escolares en Ciencias Sociales y Ciencias Naturales y su relación con los procesos de aprendizaje. El caso de Colombia. DELTA: Documentação de Estudos em Lingüística Teórica e Aplicada, 25(3), 657-663.

Moss, G. \& Chamorro, D. (2009). La enseñanza de la ciencia sin asidero en el tiempo ni en el espacio: análisis del discurso de los textos escolares. Lenguaje, 36(19), 87-115.

Moss, G., Chamorro, D., Barletta, N. \& Mizuno, J. (2013). La metáfora gramatical en los textos escolares de Ciencias Sociales en español. Onomázein Revista de Lingüística, Filología y Traducción, (28), 88-104. https://doi.org/10.7764/onomazein.28.3

Moss, G., Mizuno, J., Ávila, D., Barletta, N., Carreño, S., Chamorro, D. \& Tapia, C. (1998). Urdimbre del texto escolar: ¿Por qué resultan difíciles algunos textos? Barranquilla, Colombia: Ediciones Uninorte.

Natale, L. (2009). Lo que los manuales escolares no dicen (y los profesores debemos ayudar a comprender). DELTA: Documentação de Estudos em Lingüistica Teórica e Aplicada, 25(3), 645-656. 
Ortega, A. (2013). Lectura y escritura digital (Tesis doctoral, Universidad de Barcelona). Recuperado de http://hdl.handle.net/2445/121375

Ortega, A., Coromina, J. \& Teberosky, A. (2013). Production of Texts with Multimodal Resources by Two Groups of Primary Education Students. En J. Arnau (Ed.), Reviving Catalan at School. Challenges and Instructional Approaches (pp.120-135). Bristol, Inglaterra: Multilingual Matters.

Pereira, P. V. (2007) O ato de ler: uma análise de prática da leitura em disciplinas do ensino médio (Tesis de maestría, Universidade de Brasília). Recuperado de https://repositorio.unb.br/handle/10482/3778

Phillips Galloway, E. \& Uccelli, P. (2019a). Examining developmental relations between core academic language skills and reading comprehension for English learners and their peers. Journal of Educational Psychology. 111(1), 15-31. https://doi.org/10.1037/edu0000276

Phillips Galloway, E. \& Uccelli, P. (2019b). Beyond reading comprehension: exploring the additional contribution of core academic language skills to early adolescents' written summaries. Reading and Writing, 32, 729-759. https://doi.org/10.1007/s11145-018-9880-3

Schleppegrell, M. (2001). Linguistic features of the language of schooling. Linguistics and Education, 12(4), 431-459. https://doi.org/10.1016/S0898-5898(01)00073-0

Schleppegrell, M. (2004). The language of schooling. A functional linguistics perspective. Mahwah, NJ: Lawrence Erlbaum.

Schleppegrell, M. \& Achugar, M. (2003). Learning language and learning history: A functional linguistics approach. TESOL Journal, 12(2), 21-27. https://doi.org/10.1002/j.1949-3533.2003.tb00126.x

Schleppegrell, M., Achugar, M. \& Oteiza, T. (2004). The grammar of history: Enhancing content- based instruction through a functional focus on language. Tesol Quarterly, 38(1), 67-93. https://doi.org/10.2307/3588259

Schleppegrell, M. \& Oliveira, L. C. (2006). An integrated language and content approach for history teachers. Journal of English for Academic Purposes, 5(4), 254-268. https://doi.org/10.1016/j.jeap.2006.08.003

Sepúlveda, A. (2012). Incorporação de práticas letradas na alfabetizaçáo. Cadernos Cenpec, 2(2), 189-223. https://doi.org/10.18676/cadernoscenpec.v2i2.184

Sepúlveda, A. (2015). Aprender a estudar textos. Análise de sequências didáticas de ciências e história no $5^{\circ}$ ano do Ensino Fundamental. Informe de investigación no publicado. Laboratório de educação, São Paulo, Brasil.

Sepúlveda, A. (2017a). Avaliação do uso guiado e "semi autônomo" dos materiais Aprender a estudar textos. Informe de investigación no publicado. Laboratório de educação, São Paulo, Brasil.

Sepúlveda, A. (2017b). Discurso educativo letrado de profesoras alfabetizadoras de diferente perfil pedagógico. Educação em Revista (UFMG), 33, e141263. https://doi.org/10.1590/0102-4698141263

Sepúlveda, A., Bisognin, A. \& Paulet, N. (2017). Aprender a estudar textos. Análise de experiências de intervenção educativa em aulas de História com turmas de $4^{\circ}$ e $5^{\circ}$ ano do Ensino Fundamental. Informe de investigación no publicado. Laboratório de educação, São Paulo, Brasil.

Shulman, L. S. (1986). Those who understand: Knowledge growth in teaching. Educational Researcher, 15(2), 4-14. doi:10.3102/ 0013189X015002004

Shulman, L. S. (1987). Knowledge and teaching: Foundations of the new reform. Harvard Educational Review, 57(1), 1-22.

Shulman, L. \& Shulman, J. (2016). Como e o que os professores aprendem: uma perspectiva em transformaçấo. Cadernos Cenpec | Nova série, 6(1). https://doi.org/10.18676/cadernoscenpec.v6i1.353

Teberosky, A. (2011). Recursos lingüistics, tipogràfics, de disposició i de senyalització dels textos que ajuden a l'aprenentatge. Documento no publicado.

Teberosky, A. (2020). Palavras às professoras que ensinam a ler e escrever. Recuperado de https://labedu.org.br/wp-content/uploads/2020/11/palavrasasprofessoras.pdf

Teberosky, A., Ortega, A. \& Coromina, J. (2017). Aprendiendo a transformar un relato textual en otro multimodal. En G. Londoño Monroy \& J. L. Rodríguez Illera (Comps.), Relatos Digitales en Educación Formal y Social (pp. 59-77). Barcelona: Universidad de Barcelona, España. https://doi.org/10.1344/105.000003160

Teberosky, A. \& Sepúlveda, A. (2011, marzo). Ayudas al lector: tecnologías del pasado y del presente. Comunicación presentada en el Primer Congreso Internacional Virtual de Comprensión Lectora (CIVEL), Universidad de Málaga, España. 
Uccelli, P., Galloway, E., Barr, C., Meneses, A. \& Dobbs, C. (2015). Beyond vocabulary: Exploring cross-disciplinary academic language proficiency and its association with reading comprehension. Reading Research Quarterly, 50(3), 337-356. https://doi.org/10.1002/rrq.104

Uccelli, P., Phillips Galloway, E., Aguilar, G. \& Allen, M. (2019). Amplifying and Affirming students' voices through CALS-informed instruction, Theory Into Practice, 59(1), 75-88. https://doi.org/10.1080/00405841.2019.1665413 